\title{
Similar predictive value of six-minute walking distance and B-type natriuretic peptide in heart failure with reduced to mid-range ejection fraction
}

\author{
Angela Beatrice Scardovi ${ }^{1}$, Renata De Maria ${ }^{2}$, Gian Giacomo Galeotti ${ }^{3}$, Pompilio Faggiano ${ }^{4}$, Luca Arcari ${ }^{1,5}$, \\ Stefano Ghio ${ }^{6}$, Pier Luigi Temporelli ${ }^{7}$, Andrea Rossi ${ }^{8}$, Giovanna Magni ${ }^{* *}$, Anca Simioniuc ${ }^{3}$, Roberto Ricci ${ }^{1}$, \\ Frank Lloyd Dini ${ }^{3}$ \\ ${ }^{1}$ Cardiology Division, Santo Spirito Hospital, Rome; ${ }^{2}$ CNR Clinical Physiology Institute, ASST Great Metropolitan \\ Hospital Niguarda, Milan; ${ }^{3}$ Cardiac, Thoracic and Vascular Department, University of Pisa; ${ }^{4}$ Cardiology Department, \\ University of Brescia; ${ }^{5}$ Cardiology Unit, Clinical and Molecular Medicine Department, Sapienza University of Rome; \\ ${ }^{6}$ Cardiology Division, IRCCS Policlinico San Matteo, Pavia; ${ }^{7}$ Division of Cardiology, ICS Maugeri, IRCCS, Veruno; \\ ${ }^{8}$ Department of Medicine, Section of Cardiology, University of Verona on behalf of the Network Labs Ultrasound \\ (NEBULA) in Heart Failure Study Group; ${ }^{9}$ QBGROUP Spa, Padua, Italy; *Present address: Clinical Trials and \\ Biostatistics Unit, IRCCS Istituto Oncologico Veneto, Padua, Italy
}

\begin{abstract}
The prognostic insights of heart failure (HF) with mid-range (40-49\%) ejection fraction (HFmrEF) are not fully elucidated. We investigated whether the six-minutes walking test (6MWT) and brain natriuretic peptide (BNP) are predictive of outcome across the spectrum of LV systolic dysfunction and whether the HFmrEF cut-off impacts the risk stratification abilities of these tests. We studied 538 outpatients, aged $70 \pm 12$ years, $28 \%$ females, with stable chronic $\mathrm{HF}$ and $\mathrm{EF}<50 \%, 349$ with $\mathrm{HFmrEF}$ and 189 with HFrEF. End-points were all-cause and cardiac death. HFrEF patients were more often male, with ischemic etiology, severe symptoms, higher BNP levels, and cardiac mortality than HFmrEF
\end{abstract}

Correspondence: Angela Beatrice Scardovi, Cardiology Division, Santo Spirito Hospital, Lungo Tevere in Saxia 1, 00139 Rome, Italy. E-mail: ab.scardovi@libero.it

Contributions: ABS, RDM, FLD, contributed to the conception or design of the work; ABS, RDM, manuscript drafting; all authors contributed to the acquisition, analysis, or interpretation of data for the work; all authors critically revised the manuscript, gave final approval and agree to be accountable for all aspects of work.

Conflict of interest: The authors declare no conflict of interest.

Key words: Heart failure; mid-range ejection fraction; 6-minute walking test; BNP.

Received for publication: 11 February 2019.

Accepted for publication: 19 April 2019.

CCopyright A.B. Scardovi et al., 2019

Licensee PAGEPress, Italy

Monaldi Archives for Chest Disease 2019; 89:1045

doi: 10.4081/monaldi.2019.1045

This article is distributed under the terms of the Creative Commons Attribution Noncommercial License (by-nc 4.0) which permits any noncommercial use, distribution, and reproduction in any medium, provided the original author(s) and source are credited. subjects. During 32 (15-46) months follow-up, 123 (23\%) patients died, 95 (18\%) for cardiac causes. Cut-offs of $125 \mathrm{pg} / \mathrm{ml}$ for BNP and 360 meters for 6MWT distance were associated with lower allcause $(10 \%$ vs $38 \%, \mathrm{p}<0.001$ and $10 \%$ vs $26 \%, \mathrm{p}<0.001$, respectively) and cardiac mortality (6\% vs $36 \%, \mathrm{p}<0.001$ and $8 \%$ vs $23 \%, \mathrm{p}<0.001$, respectively). BNP (HR 2.144, 95\%CI, $1.403-$ 3.276) and 6MWT walked distance (HR 1.923, 95\%CI, 1.195-3.096) independently predicted outcome, after adjustment for age, gender, obesity, kidney dysfunction, ischemic etiology, NYHA class, unlike the $40 \%$ LVEF threshold. Model discrimination and survival differences were significant across LVEF strata. Higher BNP levels and shorter walked distance combined identified patients $(26 \%$ overall) at particularly poor prognosis in both phenotype groups. Despite differences between HFmrEF and HFrEF patients in clinical and biomarker profile, BNP levels and 6MWT walked distance retain prognostic value over the entire spectrum of LV systolic dysfunction.

\section{Introduction}

Until recently, heart failure (HF) was classified according to left ventricular ejection fraction (LVEF) into HF with reduced $(<40 \%)$ LVEF (HFrEF) and HF with preserved $(\geq 50 \%)$ LVEF (HFpEF) [1]. These entities express a diverse pathophysiological and clinical background [2], with different clinical phenotype, prognosis and response to drug therapies [3].

Although the negative prognostic value of a reduced LVEF is well established [4,5], evidences from randomised controlled trials of drug and device therapy have been derived for the $<40 \%$ threshold, i.e. the HFrEF phenotype. To address this gap in evidence the latest guidelines issued by the European Society of Cardiology include a third subtype: HF with mid-range ejection fraction (HFmrEF, LVEF 40-49\%) [6] HFmrEF has been observed to show intermediate clinical characteristics and biomarker profile between HFrEF and HFpEF [7]. Some studies reported a closer similarity in phenotype and outcomes to HFrEF [8-10] and others to HFpEF [11,12], with a modulating role of ischemic HF etiology [13], and possibly a slightly better survival in HFmrEF than in HFrEF [14]. Several clinical, laboratory, and instrumental parameters have been proposed as 
prognostic markers both for HFrEF and HFpEF [6], whereas few data are available in HFmrEF [9-13,15].

The cardiopulmonary exercise test with gas exchange measurement (CPET), a well-established tool for risk stratification in HF [16-18], is time-consuming, not well-accepted by elderly patients, and is not widely available. The six-minute walking test (6MWT) has gained popularity as a valid alternative for evaluating submaximal exercise tolerance and prognosis in clinically stable HF patients, since it is easier to perform and better tolerated $[19,20]$. Natriuretic peptides are reliable, widely used prognostic markers for patients with stable chronic HF [21,22]. Their predictive role in HF may be complementary to 6MWT [23]. To our knowledge, no previous studies addressed the combined prognostic impact of brain natriuretic peptide (BNP) and 6MWT walking distance for risk stratification of patients with HFmrEF.

Aims of the present study were to investigate whether, in a cohort of patients with chronic stable HF and systolic dysfunction (LVEF $<50 \%$ ), the 6MWT distance and BNP level are associated with outcome across the spectrum of LVEF and to verify whether the HFmrEF cut-off impacts on the risk stratification capabilities of these tests.

\section{Patients and Methods}

\section{Study population and clinical assessment}

We retrospectively includeded 538 outpatients with clinically stable chronic HF and an LV systolic dysfunction (LVEF <50\%) followed-up at HF specialist outpatient clinics, who had consecutively undergone 6MTWT between June 2008 and April 2015. Exclusion criteria were unstable angina or myocardial infarction during the previous month, and severe gait disturbances, as evaluated case by case by the clinician.

Medical history, physical examination, laboratory results and drug management of HF were registered at the time of the index echocardiogram. Venous blood was collected at the time of the visits; BNP concentrations were measured according to to the chemilluminescent microparticle immunoassay (ARCHITECT BNP, Abbott Laboratories, Abbott Park, IL, USA). The estimated glomerular filtration rate (eGFR) was calculated on the basis of the Modification of Diet in Renal Disease (MDRD) formula. Left ventricular (LV) volumes and LVEF were calculated by Doppler echocardiograpy from apical 2- and 4-chamber views using the modified Simpson's rule.

The study was approved by the local institutional review boards. Patients gave written informed consent to the use of their anonymized data for research purposes. The study was performed by the Network Labs Ultrasound (NEBULA) in Heart Failure Study Group.

\section{Six-minutes walking test}

After the first clinical and echocardiographic examination, each patient underwent a 6MWT as recommended by guidelines [24]. The tests were performed in a flat hospital corridor, and patients were instructed to walk as many meters as they could within six minutes, but not to run or jog during the test. They were also informed to stop or slow down at every moment if they experienced chest pain, intolerable dyspnea, leg cramps, dizziness, diaphoresis, and if the instructor observed a staggering gait or a pale or ashen appearance. Blood pressure was measured at the beginning and at the end of the test, while heart rate and oxygen saturation were continuously monitored during the test with a pulse oximeter.
Patients were also asked to graduate their level of dyspnea and fatigue at baseline and at the end of the test using a modified Borg scale from 0 (complete absence of dyspnea and fatigue) to 10 (very severe/maximal dyspnea and/or fatigue). During the test, a standardized encouragement was given every minute and 15 seconds before the end of the test, also informing the patients about how many minutes were left. At the end of the test the distance walked in meters, blood pressure, oxygen saturation, heart rate and the level of dyspnea and fatigue were recorded. The patients were also asked to assess whether they could walk farther.

Patients were routinely followed-up at the participating clinics. Outcome data were obtained from hospital medical records, interviews to general practitioner or contact with patients' relatives. Study end points included all-cause mortality and cardiac death, defined as death due to progressive pump failure, acute coronary syndromes or sudden.

\section{Statistical analysis}

Continuous variables are expressed as mean and standard deviation or median and interquartile range. Differences were assessed by Student's unpaired $t$-test for continuous variables or the chi-square test for categorical variables, or corresponding nonparametrical tests in case of non-normal distribution.

Probabilities of survival were estimated by the Kaplan-Meier method. Survival curves were compared by the log-rank test, stratified by LVEF level.

The association of variables with outcome was assessed by Cox regression. The proportional hazards assumption was checked visually and tested based on the scaled Schoenfeld residuals. Where applicable, continuous variables were also reclassified using clinically relevant dichotomous categorizations (e.g. obesity, stage 3 chronic kidney disease, $\mathrm{HFrEF} / \mathrm{HFmrEF}$ ); we used previously validated cut-off values for BNP $(\leq 125$ or $>125 \mathrm{pg} / \mathrm{ml})$ [25] and distance walked by the 6MWT ( $\leq 360 v s>360 \mathrm{mt})$ [26]. Variables that showed a significant association with outcomes $(p<0.1)$ were included in multivariable Cox backward stepwise models to determine those independently associated to prognosis. Model discrimination was assessed using the Harrell $C$ statistic. Significance level was set at $\mathrm{p}<0.05$.

Data were analyzed using the Statistical Package for the Social Sciences ver. 22.0 for Windows statistical software program (SPSS, Chicago, IL, USA).

\section{Results}

\section{Patients' characteristics}

The clinical characteristics of the study cohort are depicted in Table 1 . This was a stable elderly population with mild-to moderate $\mathrm{HF}$, on background guideline-recommended treatment. According to the ESC guidelines definition, 349 had HFmrEF and 189 had HFrEF. We found no differences between the two LVEF subgroups for age, duration of HF history and prevalence of diabetes, atrial fibrillation, stage 3 to 5 (eGFR $<60 \mathrm{ml} / \mathrm{min}$ ) kidney dysfunction, or use of renin-angiotensin system inhibitors. Patients with HFrEF were more commonly male, with an ischemic etiology of HF, severe symptoms and higher BNP levels and were more frequently on beta-blockers, loop diuretics and mineralocorticoid receptor antagonists than HFmrEF subjects (Table 1). Conversely patients with HFmrEF were more often women with a history of hypertension. Although HFrEF patients walked on average longer 
distances than HFmrEF, no differences were observed when values were expressed as percent of predicted. At entry into the study a subgroup of HFmrEF patients had an implantable cardioverter defibrillator $(n=46,13 \%)$ without or with biventricular pacing $(6 \%)$ suggesting that at the time of our first assessment they had recovered LVEF from $<35 \%$ to mid-range.

\section{Outcome}

During a median follow-up of 32 [15-46] months, overall 123 (23\%) patients died, 95 (18\%) of cardiac causes. Cardiac death was significantly more common than non-cardiac mortality among HFrEF patients (Table 1). Five-year unadjusted survival rates were higher in HFmrEF than in HFrEF both for all-cause and cardiac mortality $(\mathrm{p}=0.005)$ (Figure 1$)$

By univariable analysis (Table 2), older age, male gender, lower eGFR, longer duration of HF, lower BMI, an ischemic etiology, NYHA class III, lower distance walked, higher BNP levels, lower LVEF were associated to all-cause and cardiac mortality, while diabetes also predicted cardiac death.

Multivariable Cox proportional hazards models (Table 3) identified as independent predictors of outcome older age, male gender, stage $3 \mathrm{CKD}$, a non-obese phenotype, ischemic etiology of HF, NYHA class III, BNP levels $>125 \mathrm{pg} / \mathrm{ml}$ and $\leq 360$ meters walked in $6 \mathrm{~min}$. A HFmrEF phenotype per se was not associated with either cardiac or all-cause mortality (HR $0.886,95 \%$ CI 0.552 1.423 and HR $0.8196,95 \%$ CI $0.535-1.224$, respectively) after covariate adjustement. Models C statistic (Table 3) for both allcause and cardiac mortality was similarly high in HFrEF $(0.801$ (95\% 0.740-0.862) and 0.833 (95\% CI 0.778-0.888), respectively) and HFmrEF patients $(0.781(95 \% 0.716-0.846)$ and $0.79(95 \% \mathrm{CI}$ $0.723-0.857$, respectively).

Among patients with BNP levels $\leq 125 \mathrm{pg} / \mathrm{ml} v s$ those with BNP concentrations $>125 \mathrm{pg} / \mathrm{ml}$ (207 subjects, 38\%) all-cause and cardiac mortality rates were $10 \%$ vs $38 \%(\mathrm{p}<0.001)$ and $6 \%$ vs $36 \%$ $(\mathrm{p}<0.001)$, respectively. Among patients who walked $\leq 360 \mathrm{mt}$ vs those who walked $>360$ meters (341 subjects, 63\%) all-cause and cardiac mortality rates were $10 \%$ vs $26 \%(\mathrm{p}<0.001)$ and $8 \%$ vs $23 \%$ $(p<0.001)$, respectively. Survival differences were significant across LVEF strata both for BNP (Figure 2) and distance walked (Figure 3). The combination of higher BNP levels and shorther walked distance identified patients ( $26 \%$ overall) at particulary poor prognosis in both phenotype groups (Figure 4).

Table 1. Clinical characteristics of the study population

\begin{tabular}{|c|c|c|c|c|}
\hline & $\begin{array}{c}\text { All } \\
\mathrm{n}=538\end{array}$ & $\begin{array}{l}\text { HFrEF } \\
n=189\end{array}$ & $\begin{array}{c}\text { HFmrEF } \\
\mathrm{n}=349\end{array}$ & p \\
\hline Age, years & $70 \pm 12$ & $70 \pm 12$ & $70 \pm 11$ & 0.886 \\
\hline Age $\geq 70$ years & $293(54)$ & $107(57)$ & $186(53)$ & 0.470 \\
\hline Female (n, \%) & $151(28)$ & $38(20)$ & $113(32)$ & 0.003 \\
\hline Hypertension (n, \%) & $434(81)$ & $137(73)$ & $297(85)$ & 0.001 \\
\hline Diabetes (n, \%) & $146(27)$ & $56(30)$ & $90(26)$ & 0.360 \\
\hline BMI kg/m² & $27 \pm 4.7$ & $26.5 \pm 4.2$ & $27.3 \pm 4.9$ & 0.074 \\
\hline $\mathrm{BMI} \geq 30 \mathrm{~kg} / \mathrm{m}^{2}(\mathrm{n}, \%)$ & $124(23)$ & $38(20)$ & $87(25)$ & 0.241 \\
\hline $\mathrm{eGFR} \mathrm{ml/min} / 1.73 \mathrm{~m}^{2}$ & $64 \pm 24$ & $62 \pm 25$ & $64 \pm 23$ & 0.377 \\
\hline Stage 3 renal dysfunction* (n, \%) & $270(50)$ & $98(52)$ & $172(49)$ & 0.589 \\
\hline Atrial fibrillation (n, \%) & $104(19)$ & $37(20)$ & $67(19)$ & 0.909 \\
\hline Duration of heart failure, years & $5(3)$ & $5(3)$ & $5(2)$ & 0.10 \\
\hline Ischemic etiology (n, \%) & $200(37)$ & $93(49)$ & $107(31)$ & 0.0001 \\
\hline NYHA class III (n, \%) & $126(23)$ & $52(27)$ & $74(21)$ & 0.11 \\
\hline LVEF\% & $41 \pm 8$ & $31 \pm 6$ & $46 \pm 3$ & \\
\hline BNP pg/ml (median IQR) & $100(68-223)$ & $190(89-611)$ & $90(61-140)$ & 0.001 \\
\hline Distance walked meters & $335 \pm 132$ & $355 \pm 132$ & $325 \pm 115$ & 0.010 \\
\hline Distance walked \% predicted & $70 \pm 24$ & $73 \pm 25$ & $69 \pm 24$ & 0.096 \\
\hline \multicolumn{5}{|l|}{ Treatment } \\
\hline Loop diuretics (n, \%) & $443(82)$ & $182(96)$ & $261(75)$ & 0.001 \\
\hline RAS-inhibitors (n, \%) & $498(93)$ & $173(91)$ & $325(93)$ & 0.496 \\
\hline MR antagonists (n, \%) & $304(56)$ & $171(90)$ & $133(38)$ & 0.001 \\
\hline Betablockers (n, \%) & $427(79)$ & $162(86)$ & $265(76)$ & 0.007 \\
\hline CRT (n, \%) & $76(14)$ & $56(30)$ & $20(6)$ & 0.001 \\
\hline $\operatorname{ICD}(\mathrm{n}, \%)$ & $143(27)$ & $97(51)$ & $46(13)$ & 0.001 \\
\hline Cause of death & & & & 0.001 \\
\hline Cardiac & $95(18)$ & $53(28)$ & $42(12)$ & \\
\hline Non-cardiac & $28(5)$ & $7(4)$ & $21(6)$ & \\
\hline
\end{tabular}

Data are number (frequency percent), mean (SD) or median IQR; BMI, body mass index; BNP, brain natriuretic peptide; CRT, cardiac resynchronization therapy; CV cardiovascular; ICD, implantable cardioverter defibrillator; LVEF, left ventricular ejection fraction; MR, mineralocorticoid receptor; NYHA, New York Heart Association; RAS, renin angiotensin system; *eGFR <60 ml/min/1.73 m². 


\section{Discussion}

To our knowledge, this is the first study to assess the prognostic significance of the 6MWT walking distance and BNP values across the HFrEF-HFmrEF spectrum. We found that the predictive value of these two parameters is similar in both phenotypes and independent of other common prognostic markers such as age, gender, ischemic etiology, advanced NYHA class, obesity, and CKD. Conversely, in patients with chronic stable $\mathrm{HF}$ and $\mathrm{LVEF}<50 \%$, although LVEF as a continuous value retains a significant association with outcome, the HF phenotype (HFrEF vs HFmrEF) has no independent prognostic impact, after adjustement for relevant covariates.

The new guidelines classification of HFmrEF has stimulated a wealth of observational studies into the clinical characteristics, biomarker profile and prognosis of this novel phenotype, with many
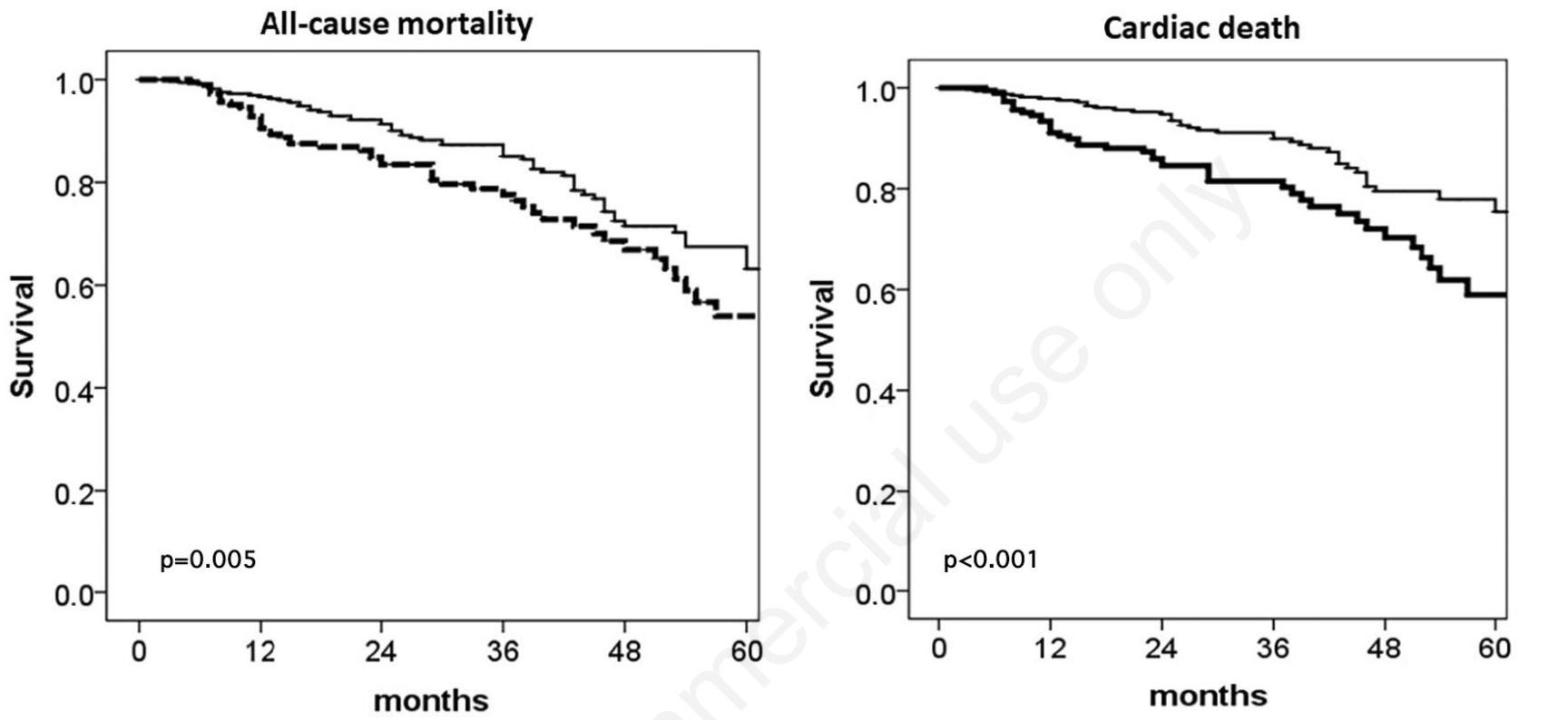

Figure 1. Survival curves for HFmrEF (light line) and HFrEF (thick line).
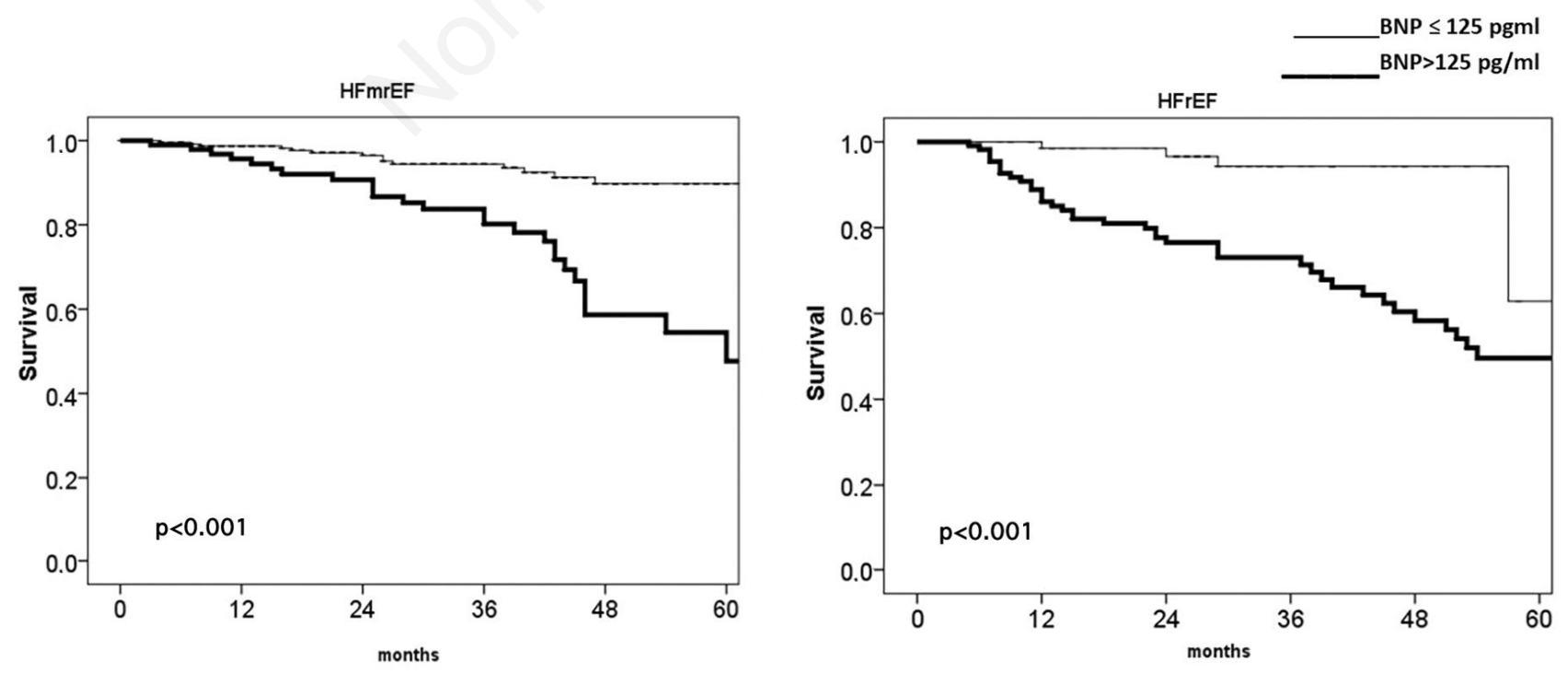

Figure 2. Survival according to a BNP cut-off $>25$ (thick line) or $\leq 125 \mathrm{pg} / \mathrm{ml}$ (light line) in patients with HFmrEF (left panel) or HFrEF (right panel). 
contradictory findings [7-15]. In our series we observed a similar comorbidity burden, with differences in gender ratio and prevalent etiology, a better crude survival and a lower cardiac to non-cardiac death ratio for HFmrEF patients when compared to the HFrEF group.

$6 \mathrm{MWT}$ is a simple, feasibile test that correlates consistently with CPET variables and has shown similar prognostic power for stable ambulatory outpatients $[19,20,23]$. We confirmed that a decreasing 6MWT distance is associated to a poor prognosis, with a previously validated threshold of 360 meters conferring an approximately twofold risk of all-cause mortality and three-fold risk of cardiac death. Our cohort consisted mainly of mild-to-moderate chronic HF patients and in this subset the 6MWT might be considered more as a submaximal rather than a maximal test. However, the independent predictive value of the $6 \mathrm{MWT}$ distance for poor outcome supports its use as objective measurement of functional capacity in clinical practice for risk stratification in an elderly population.

The prognostic relevance of NP in chronic HF is well established [21]. BNP was validated in the Valsartan HEart failure Trial as a powerful independent predictor of poor outcome even taking into account demographic, clinical and echocardiographic confounders [26]. While most of the studies on the prognostic role of NP predominantly involved HFrEF patients, there are also data in patients with slightly reduced (40-50\%) or actually preserved LVEF (HFpEF). NT-proBNP levels have been reported to be smilarly elevated in HFrEF and HFmrEF, and significantly higher in both these groups than in HFpEF [10]; targeting therapy towards a reduction in NP levels resulted in improved survival free of HF hospitalizations in HFrEF and HFmrEF, but not in HFpEF [10]. Conversely, others [27]

Table 2. Univariable predictors of outcome

\begin{tabular}{|c|c|c|c|c|c|c|c|c|}
\hline & & ll-cau: & rtality & & & Card & ath & \\
\hline & HR & $95 \%$ & CI & p & HR & $95 \%$ & CI & p \\
\hline Age (per 5 years) & 1.310 & 1.188 & 1.445 & $<0.001$ & 1.284 & 1.142 & 1.444 & $<0.001$ \\
\hline Male sec & 2.123 & 1.313 & 3.434 & 0.002 & 2.567 & 1.427 & 4.620 & 0.002 \\
\hline Hypertension & 1.217 & 0.737 & 2.008 & 0.443 & 1.456 & 0.794 & 2.670 & 0.224 \\
\hline Diabetes & 1.355 & 0.927 & 1.982 & 0.117 & 1.601 & 1.048 & 2.448 & 0.030 \\
\hline BMI kg/m² (per unit) & 0.941 & 0.904 & 0.984 & 0.003 & 0.942 & 0.900 & 0.986 & 0.010 \\
\hline BMI $<30 \mathrm{~kg} / \mathrm{m}^{2}$ & 2.399 & 1.370 & 4.201 & 0.002 & 2.396 & 1.271 & 4.515 & 0.007 \\
\hline eGFR per ml/min $/ 1.73 \mathrm{~m}^{2}$ & 0.978 & 0.969 & 0.987 & $<0.001$ & 0.969 & 0.958 & 0.980 & $<0.001$ \\
\hline $\mathrm{eGFR}<60 \mathrm{ml} / \mathrm{min} / 1.73 \mathrm{~m}^{2}$ & 2.376 & 1.598 & 3.533 & $<0.001$ & 2.859 & 1.784 & 4.583 & $<0.001$ \\
\hline Atrial fibrillation & 1.398 & 0.928 & 2.107 & 0.109 & 1.345 & 0.838 & 2.159 & 0.219 \\
\hline HF duration (year) & 1.122 & 1.062 & 1.185 & $<0.001$ & 1.161 & 1.092 & 1.234 & $<0.001$ \\
\hline Ischemic etiology & 2.292 & 1.597 & 3.291 & $<0.001$ & 2.632 & 1.732 & 3.998 & $<0.001$ \\
\hline NYHA class III vs I-II & 2.238 & 1.561 & 3.209 & $<0.001$ & 2.572 & 1.714 & 3.859 & $<0.001$ \\
\hline LVEF (per 5 pp) & 0.865 & 0.784 & 0.954 & $<0.001$ & 0.803 & 0.728 & 0.885 & $<0.001$ \\
\hline LVEF $<40$ vs $\geq 40 \%$ & 1.647 & 1.113 & 2.353 & 0.006 & 2.148 & 1.427 & 3.233 & $<0.001$ \\
\hline BNP (per 25 pg/ml) & 1.006 & 1.004 & 1.007 & $<0.001$ & 1.007 & 1.005 & 1.008 & $<0.001$ \\
\hline $\mathrm{BNP}>125 \mathrm{pg} / \mathrm{ml}$ & 3.526 & 2.369 & 5.246 & $<0.001$ & 5.347 & 3.226 & 8.863 & $<0.001$ \\
\hline Meters walked (per 10) & 0.961 & 0.942 & 0.98 & $<0.001$ & 0.961 & 0.942 & 0.980 & $<0.001$ \\
\hline Meters walked <360 & 2.589 & 1.642 & 4.084 & $<0.001$ & 2.985 & 1.739 & 5.124 & $<0.001$ \\
\hline
\end{tabular}

BMI, body mass index; BNP, brain natriuretic peptide; eGFR, estimated glomerular filtration rate; LVEF, left ventricular ejection fraction; NYHA, New York Heart Association.

Table 3. Multivariable models of all-cause and cardiac mortality.

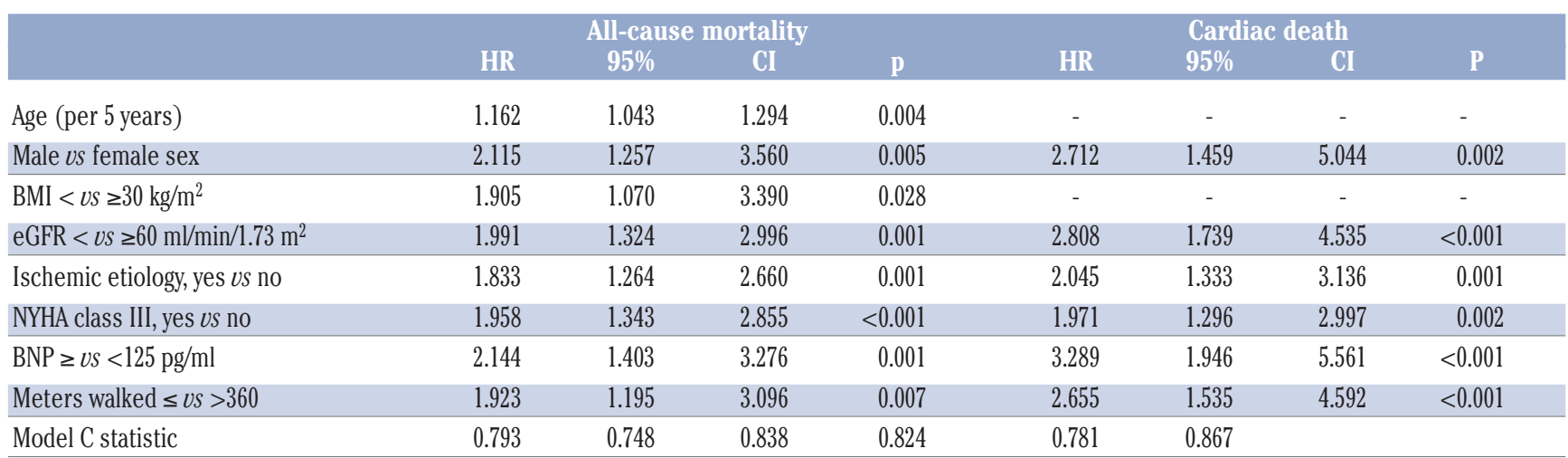

Variables entered in the models: age, sex, diabetes (cardiac death only), BMI, eGFR, ischemic etiology of HF, HFmrEF or HFrEF, duration of heart failure, NYHA class, BNP, walked distance. BMI, body mass index; BNP, brain natriuretic peptide; eGFR, estimated glomerular filtration rate; NYHA, New York Heart Association. 
reported comparably lower NT-proBNP levels in HFmrEF and $\mathrm{HFpEF}$ than in $\mathrm{HFrEF}$, and suggested that the predictive performance of NP for combined end points of mortality and HF admission may be higher in HFmrEF than in HFpEF.

In our large group of patients with systolic dysfunction (LVEF $<50 \%$ ), the prognostic relevance of BNP was similar in HFmrEF and HFrEF patients, despite higher values in the latter group. The finding of a stronger predictive value of BNP levels for cardiac death than for all-cause mortality is consistent with the interpretation provided in the Swedish Heart Failure Registry analysis [28]: non-cardiovascular mortality risk increases with higher LVEF and cardiovascular-to-non-cardiovascular event ratio raises with increasing NT-proBNP concentrations.

Combining BNP values with the 6MWT might provide a simple and efficient method for the assessment of long-term prognosis of both HFmrEF and HFrEF patients. BNP levels are more closely
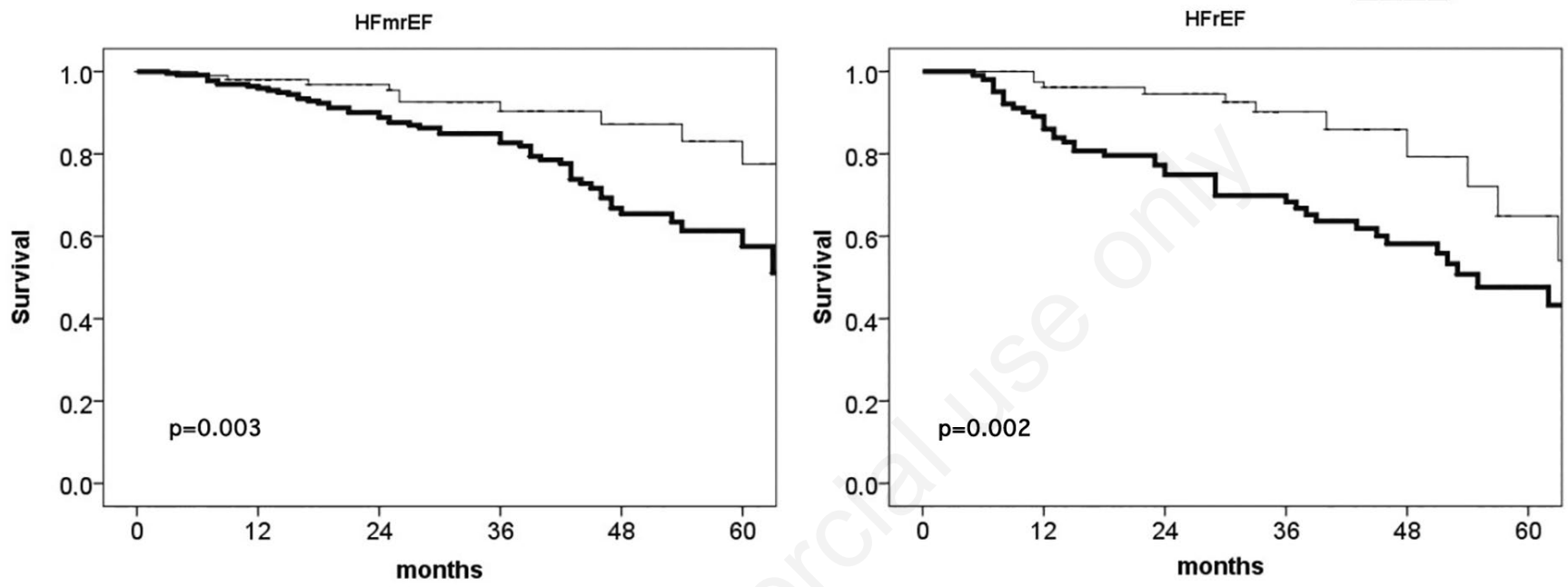

Figure 3. Survival according to a $6 \mathrm{MWT}$ distance walked cut-off $\leq 360$ (thick line) or $>360$ meters (light line) in patients with HFmrEF (left panel) or HFrEF (right panel).
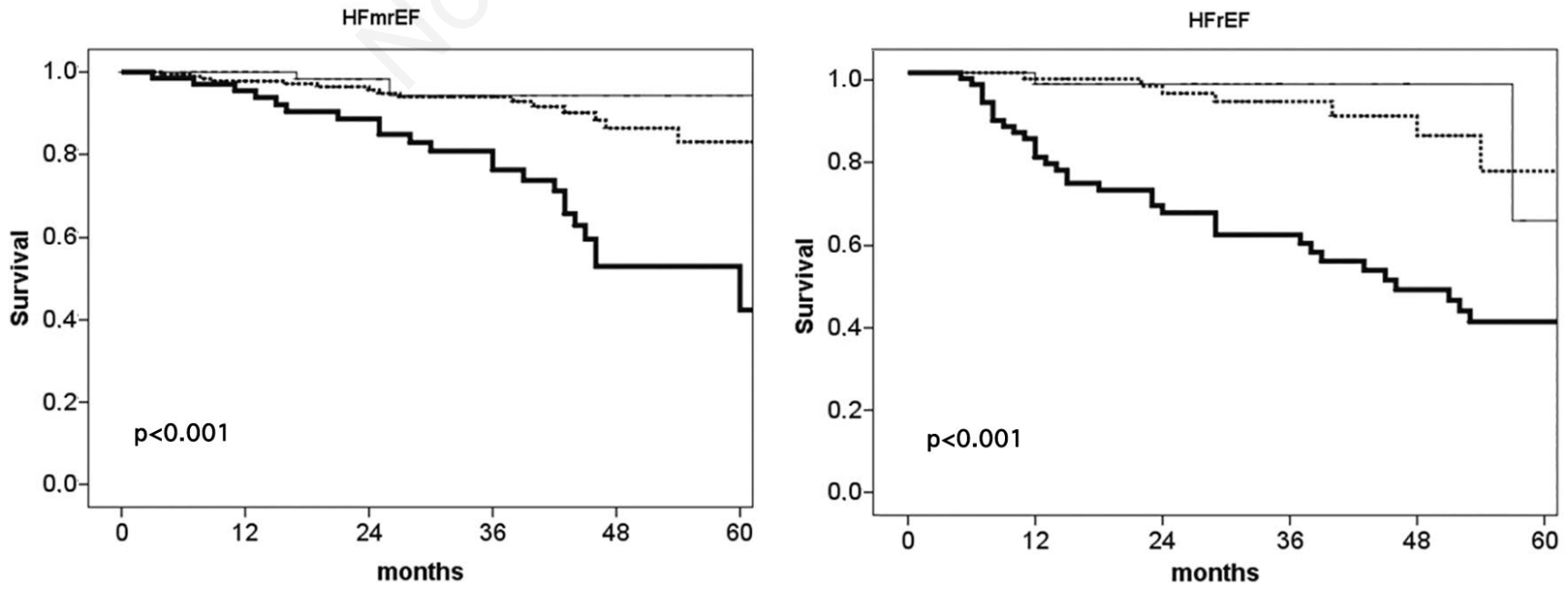

Figure 4. Survival according to presence of a BNP $\leq 125 \mathrm{pg} / \mathrm{ml}$ and a $6 \mathrm{MWT}$ distance $>360$ meters (light line), either a $\mathrm{BNP}>125 \mathrm{pg} / \mathrm{ml}$ or a walked distance $\leq 360$ meters (hatched line), or the combination of BNP $>125 \mathrm{pg} / \mathrm{ml}$ and a walked distance $\leq 360$ meters (thick line in patients with HFmrEF (left panel) or HFrEF (right panel). 
related to central hemodynamic parameters and indices of cardiac function [22]. Conversely, exercise tolerance also depends on the severe structural and functional abnormalities observed in the skeletal muscle in HF, with an excess catabolism that culminates in cardiac cachexia in advanced HF. This interpretation is further supported by the independent protective role of obesity in our series, in agreement with the long-described "obesity paradox" in patients with overt HF [29]. Hence BNP and walking distance can represent different, but complementary, facets of the complexity of the HF syndrome. The $6 \mathrm{MWT}$, being a more a global test, rather than just a cardiovascular performance test, is probably able to investigate the "syndromic" aspects of CHF. Consequently, the combined evaluation of these two indicators of disease severity is a simple way to simultaneously assess different important features of the disease. Actually, the coexistence of higher BNP levels and shorter walked distance portends a distinctly poor prognosis.

HFmrEF is a recently defined entity, and few studies exist on its prognostic significance compared to HFpEF and HFrEF. Outcome for HFmrEF patients has alternatively been reported to be similar to HFpEF $[12,27]$ or HFrEF $[8,9]$ or both $[10,11]$ or intermediate $[13,14]$. These conflicting results may be justified by the vast heterogeneity of the HF syndrome within the studied populations, as supported by the modulating role of ischemic heart disease on mortality in HFmrEF [13]. Furthermore, HFmrEF is possibly an even more heterogeneous subgroup, comprising patient with a stable moderate LVEF reduction, as well as patients who transitioned from HFpEF or HFrEF, occurrences that carry a different prognosis [7,13,30,31]. Based on our results, differentiating between HFrEF and HFmrEF does not provide significant prognostic information in patients with chronic stable HF and impaired LVEF, after adjustment for other relevant predictors of survival. Moreover, model discrimination overlapped in both HFmrEF and HFrEF subgroups, highlighting the value of NP and 6MWT for risk stratification in systolic dysfunction.

\section{Limitations}

Our multicenter observational study carries the inherent limitations of this design. Patients were enrolled at varying distances from the first onset of HF, hence we were unable to distinguish the subset of patients with previously reduced or recovered EF. Our analysis correlates baseline assessments with hard mortality endpoints, but does not provide longitudinal data, hence we cannot speculate about changes in HF phenotype over time and their relation with prognosis.

Some differences in drug therapy were observed between groups. Consistently with guidelines recommendations, HFrEF patients received in very high proportions and significantly more often than HFmrEF patients, beta-blockers, loop diuretics and mineralocorticoid receptor antagonists, but not renin-angiotensin system inhibitors, with respect. These variances might highlight a different perception of disease severity or, in the case of diuretics, deprescribing attempts for long-standing stability, but the lack of longitudinal data does not allow firm conclusions. However, lower drug therapy uptake did not translate in worse outcomes (Figure 1).

Our patients had mild-to-moderate chronic stable HF and therefore our findings cannot be extended to patients with more severe or acutely decompensated HF. Our aim was to assess the predictive value of two tests BNP and 6MWT after adjustment for relevant covariates. To avoid model overfitting, we limited adjustment to only some of the many variables that have been associated with outcome in HF with LVEF $<50 \%$, although we checked that representative variables for important domains in $\mathrm{HF}$ assessment were included.

\section{Conclusions}

Our study confirms differences between HFmrEF and HFrEF patients in clinical and biomarker profile and long-term outcome, and points out that BNP levels and distance walked retain a similar prognostic value across phenotypes in patients with reduced LVEF.

\section{References}

1. McMurray JJ, Adamopoulos S, Anker SD, et al. ESC Guidelines for the diagnosis and treatment of acute and chronic heart failure 2012: The Task Force for the Diagnosis and Treatment of Acute and Chronic Heart Failure 2012 of the European Society of Cardiology. Developed in collaboration with the Heart Failure Association (HFA) of the ESC. Eur Heart J 2012;3:1787-847.

2. Paulus WJ, Tschöpe C. A novel paradigm for heart failure with preserved ejection fraction: comorbidities drive myocardial dysfunction and remodeling through coronary microvascular endothelial inflammation. J Am Coll Cardiol 2013;62:263-71.

3. Ferrari R, Böhm M, Cleland JG, et al. Heart failure with preserved ejection fraction: uncertainties and dilemmas. Eur J Heart Fail 2015;17:665-71.

4. Bristow MR, Kao DP, Breathett KK, et al. Structural and functional phenotyping of the failing heart: is the left ventricular ejection fraction obsolete? JACC Heart Fail 2017;5:772-781.

5. Rahimi K, Bennett D, Conrad N at al. Risk prediction in patients with heart failure: a systematic review and analysis. JACC Heart Fail 2014;2:440-6.

6. Ponikowski P, Voors AA, Anker SD, at al. 2016 ESC Guidelines for the diagnosis and treatment of acute and chronic heart failure: The Task Force for the diagnosis and treatment of acute and chronic heart failure of the European Society of Cardiology (ESC)Developed with the special contribution of the Heart Failure Association (HFA) of the ESC. Eur Heart J 2016;37:2129-200.

7. Nauta JF, Hummel YM, van Melle JP, et al. What have we learned about heart failure with mid-range ejection fraction one year after its introduction? Eur J Heart Fail 2017;19:1569-73.

8. Farré N, Lupon J, Roig E, et al. Clinical characteristics, oneyear change in ejection fraction and long-term outcomes in patients with heart failure with mid-range ejection fraction: a multicentre prospective observational study in Catalonia (Spain). BMJ Open 2017;7:e018719.

9. Bhambhani V, Kizer JR, Lima JAC, et al. Predictors and outcomes of heart failure with mid-range ejection fraction. Eur J Heart Fail 2018;20:651-659.

10. Rickenbacher P, Kaufmann BA, Maeder MT, et al. Heart failure with mid-range ejection fraction: a distinct clinical entity? Insights from the Trial of Intensified versus standard Medical therapy in Elderly patients with Congestive Heart Failure (TIME-CHF). Eur J Heart Fail 2017;19:1586-96.

11. Delepaul B, Robin G, Delmas C, et al. Who are patients classified within the new terminology of heart failure from the 2016 ESC guidelines? ESC Hear Fail 2017;4:99104.

12. Tsuji K, Sakata Y, Nochioka K, et al. Characterization of heart 
failure patients with mid-range left ventricular ejection fractiona report from the CHART-2 Study. Eur J Heart Fail 2017;19: 1258-69.

13. Koh AS, Tay WT, Teng THK, et al. A comprehensive population-based characterization of heart failure with midrange ejection fraction. Eur J Heart Fail 2017;19:1624-34.

14. Altaie S, Khalife W. The prognosis of mid-range ejection fraction heart failure: a systematic review and meta-analysis. ESC Heart Failure 2018;5:1008-1016

15. Chioncel O, Lainscak M, Seferovic PM, et al. Epidemiology and one-year outcomes in patients with chronic heart failure and preserved, mid-range and reduced ejection fraction: an analysis of the ESC Heart Failure Long-Term Registry. Eur J Heart Fail 2017; 19:1574-85.

16. Scardovi AB, De Maria R, Coletta C, et al. Multiparametric risk stratification in patients with mild to moderate chronic heart failure. J Card Fail 2007;13:445-51.

17. Agostoni $\mathrm{P}$, Corrà U, Cattadori $\mathrm{G}$, et al. Metabolic exercise test data combined with cardiac and kidney indexes, the MECKI score: a multiparametric approach to heart failure prognosis. Int J Cardiol 2013;167:2710-8.

18. Sato T, Yoshihisa A, Kanno Y, et al. Cardiopulmonary exercise testing as prognostic indicators: Comparisons among heart failure patients with reduced, mid-range and preserved ejection fraction. Eur J Prev Cardiol 2017;24:1979-87 .

19. Faggiano P, D'Aloia A, Gualeni A, et al. The 6 minute walking test in chronic heart failure: indications, interpretation and limitations from a review of the literature. Eur J Heart Fail 2004;6:687-91.

20. Forman DE, Fleg JL, Kitzman DW, et al. 6-min walk test provides prognostic utility comparable to cardiopulmonary exercise testing in ambulatory outpatients with systolic heart failure. J Am Coll Cardiol 2012;60:2653-61.

21. Oremus M, Don-Wauchope A, McKelvie R, et al. BNP and NT-
proBNP as prognostic markers in persons with chronic stable heart failure. Heart Fail Rev 2014;19:471-505.

22. Masson S, Latini R, Anand IS, et al. Prognostic value of changes in N-terminal pro-brain natriuretic peptide in Val-HeFT (Valsartan Heart Failure Trial). J Am Coll Cardiol 2008;52:997-1003.

23. Hogenhuis J, Jaarsma T, Voors AA, et al. Correlates of B-type natriuretic peptide and 6-min walk in heart failure patients. Int J Cardiol 2006;108:63-7.

24. ATS Committee on Proficiency Standards for Clinical Pulmonary Function Laboratories. ATS Statement. Am J Respir Crit Care Med 2002;166:111-7. doi:10.1164/ajrccm.166.1.at1102.

25. Ingle L, Cleland JG, Clark AL. The long-term prognostic significance of 6-minute walk test distance in patients with chronic heart failure. Biomed Res Int 2014;2014:505969.

26. Latini R, Masson S, Anand I, et al. The comparative prognostic value of plasma neurohormones at baseline in patients with heart failure enrolled in Val-HeFT. Eur Heart J 2004;25:292-9.

27. Moliner P, Lupón J, Barallat J, et al. Bio-profiling and bioprognostication of chronic heart failure with mid-range ejection fraction. Int J Cardiol 2018;257:188-92.

28. Savarese G, Orsini N, Hage C, et al. Utilizing NT-proBNP for eligibility and enrichment in trials in HFpEF, HFmrEF, and HFrEF. JACC Heart Fail 2018;6:246-56.

29. Lavie CJ, Sharma A, Alpert MA, et al. Update on Obesity and Obesity Paradox in Heart Failure. Prog Cardiovasc Dis 2016;58:393-400.

30. Rastogi A, Novak E, Platts AE, et al. Epidemiology, pathophysiology and clinical outcomes for heart failure patients with a mid-range ejection fraction. Eur J Heart Fail 2017;19: 1597-605.

31. Nadruz W, West E, Santos M, et al. Heart failure and midrange ejection fraction: implications of recovered ejection fraction for exercise tolerance and outcomes. Circ Heart Fail 2016;9:e002826. 